\title{
Minimum and Maximum Time-Localized Complex-Valued Wavelets for Scattering Problems
}

Jeng-Long Leou ${ }^{1 *}$, Jiunn-Ming Huang ${ }^{2}$, Shyh-Kang Jeng ${ }^{1}$ and Hsueh-Jyh $\mathrm{Li}^{1}$

${ }^{1}$ Dept. of Electrical Engineering, National Taiwan University, Taipei, Taiwan, ROC

${ }^{2}$ Dept. of Comm. Eng., National Chiao Tung University, Hsinchu, Taiwan, ROC

\section{Introduction}

Publications on numerical methods of electromagnetic filed problems have shown a marked preference for real-valued wavelets [1]-[2]. This is probably because the original wavelets, which were widely used for signal/image processing, were primarily real valued. Among these commonly used wavelets, especially, real-valued Daubechies wavelets are concerned most frequently owing to their finite support and orthogonality properties [3]. Nevertheless, it does not mean that they are the only choice to solve the problem. They are just one genus of wavelets within the Daubechies family. Various genus of wavelets can be constructed, real-valued or complexvalued, by means of adjusting the selection strategy of zeros in the Daubechies polynomial [3].

As is generally recognized, in real-valued wavelet family, symmetric or antisymmetric, compactly supported and orthogonal scaling functions and wavelets are not available [3] except for Haar wavelets. One of the properties mentioned above must be released if the other two properties are required. In the meantime, orthogonality and finite support wavelets are most preferred and lead to asymmetric real-valued wavelets. However, this is not to say symmetric property is not important for solving electromagnetic problems. The purpose here is to explore a little further into the symmetric or antisymmetric wavelets with compact supports and orthogonal behavior.

Since there are many wavelets genus can be obtained, either real-valued or complex-valued, we need a reasonable criterion to choose the most suitable basis for the application at hand. In this paper, a new selection criterion based on the time-localization measure of scaling functions and wavelets is proposed to investigate the relationship between the localization of wavelets and the sparsity of the resultant MoM matrix equation.

\section{Wavelet construction and selection criterion}

Consider the Daubechies polynomial [3]

$$
P(z)=\sum_{k=0}^{p-1}\left(\begin{array}{c}
p-1+k \\
k
\end{array}\right)\left(\frac{-z^{-1}+2-z}{4}\right)^{k}
$$

where $p$ is the vanishing moment and the factorization of $P(z)$ into two equal power factors is not unique. Different root selection in factorization leads to different genus of wavelets. For those genus of wavelets with real-valued coefficients, the complex conjugate $z^{*}$ is also a root when $z$ is a root. A famous choice is to have all zeros inside and on the unit circle. That gives the well-known real-valued Daubechies wavelets.

0-7803-5639-X/99/\$10.00 O1999 IEEE. 
If orthogonality and strict symmetry are required, the constraint of real-valued $h(n)$ must be released. A set of complex-valued wavelets and scaling function can be constructed by selecting roots of $P(z)$ for $N=2+4 m[4]$, where $m$ is a positive integer. The choice is to include the pair $\left(z_{i}, 1 / z_{i}\right)$ or its conjugate $\left(z_{i}^{*}, 1 / z_{i}^{*}\right)$ to a factor. Furthermore, there are $2^{m}$ permutation of roots and therefore $2^{m}$ genus of symmetric complex-valued wavelets for each $N$. Every permutation will get a symmetric $h(n)$.

Because different factorization of $P(z)$ does not change the spectral magnitude of the corresponding scaling function $\phi(t)$, but only its phase. Hence, instead of the spectral domain approach, an alternative approach in the time domain is proposed. We define the time domain root mean square (RMS) duration $T$ and the mean value $t_{m}$ as follows:

$$
\begin{aligned}
t_{m} & =\frac{1}{E_{x}} \int_{-\infty}^{+\infty} t|x(t)|^{2} d t \\
T & =2 \sqrt{\frac{\pi}{E_{x}} \int_{-\infty}^{+\infty}\left(t-t_{m}\right)^{2}|x(t)|^{2} d t}
\end{aligned}
$$

where $E_{x}$ is the energy of the signal $x(t)$. Our selection criterion is to search for a subset of wavelet bases with the minimum or the maximum time-localization measure within the Daubechies family for a given vanishing moment.

\section{Numerical results}

The filter coefficients $h(n)$ corresponding to $\phi(t)$ for the minimum and the maximum localized complex-valued wavelets with maximal vanishing moments $p=7$ are presented in Table 1 , where only half of $h(n)$ is listed owing to the symmetry property of $h(n)$. Figures 1 and 2 give plots of the complex-valued scaling functions and mother wavelet corresponding to Table 1 , where solid line and dotted line are corresponding to the the real part and imaginary part of these basis functions, respectively. The symmetric/antisymmetric properties of these basis functions can be found.

To study the sparsification ability of these wavelets constructed by our localization measure, we computed the cases of time-harmonic scattering from square cylinder (Fig. 3(a)) and circular cylinder (Fig. 3(b)) with $N=512$, and the element threshold adjusted to maintain a solution error about $5 \times 10^{-5}$ and $10^{-2}$ for comparison. Here, the independent variable $t$ of wavelets is related the perimeter of cylinders. Figure 4 shows the results of compression ratio vs. different vanishing moment for minimum localized complex-valued Daubechies wavelet (Min $\mathrm{CD})$, maximum localized complex-valued Daubechies wavelet (Max CD) and real-valued Daubechies wavelet (RD). As this figure indicates, wavelets with minimum localized width will render a more sparse matrix then both the maximum localized wavelet and real-valued wavelet do, especially for scatterer with corners (Fig.4(a)). For objects with smooth circumference (Fig.4(b)), the difference of compression ratio reduces, and the results of real-valued wavelets are similar to the minimum localized wavelets. 


\section{Conclusions}

Localization property of wavelets in the $t$-domain is one of the important mechanism to sparsify the MoM matrix. Wavelets with a smaller RMS width are more suitable for scatterers with corners. For smooth objects, the localization behavior is not so important.

\section{Reference}

[1] B. Z. Steinberg and Leviatan, "On the use of wavelet expansions in the method of moments," IEEE Trans. Antennas Propagat., vol. 41, No. 5, pp. 610-619, May 1993.

[2] R. L. Wagner and W. C. Chew, "A study of wavelets for the solution of electromagnetic integral equations," IEEE Trans. Antennas Propagat., vol. 43, pp. 802-810, Aug. 1995.

[3] W. Lawton, "Application of complex valued wavelet transform to subband decomposition," IEEE Trans. Signal Processing, vol. 41, pp. 3566-3568, Dec. 1993.

[4] I. Daubechies, "Ten Lectures on Wavelets." Philadelphia: SIAM, 1992.

Table 1: The filter coefficients $h(n)$ for the compactly supported wavelets with minimum or maximum time-localization width. The $h(n)$ is symmetry and $h(N / 2-n)=h(N / 2+n-1)$ for $n=0, \cdots, N / 2-1$.

\begin{tabular}{|c|rr||rr|}
\hline & \multicolumn{2}{|c|}{ Minimum localized } & \multicolumn{2}{c|}{ Maximum localized } \\
\hline $\mathbf{n}$ & real part & imaginary part & real part & imaginary part \\
\hline 0 & -0.00478501383501 & 0.00215429535695 & 0.00491201491997 & -0.00184647104289 \\
1 & -0.00041728660290 & -0.00092685627419 & -0.00541112994375 & -0.01439478356292 \\
2 & 0.03214578918237 & -0.02703224210922 & -0.07010899963501 & -0.00790400013796 \\
3 & -0.01294647838617 & -0.03415969588109 & -0.05643777882023 & 0.11693769460129 \\
4 & -0.11982020707840 & 0.02730297770393 & 0.18723481730045 & 0.25963126139522 \\
5 & 0.13403784165899 & 0.05080134014108 & 0.36763850555869 & 0.04759280947681 \\
6 & 0.67889213624767 & -0.01813981893746 & 0.27927935180643 & -0.40001651072956 \\
\hline
\end{tabular}
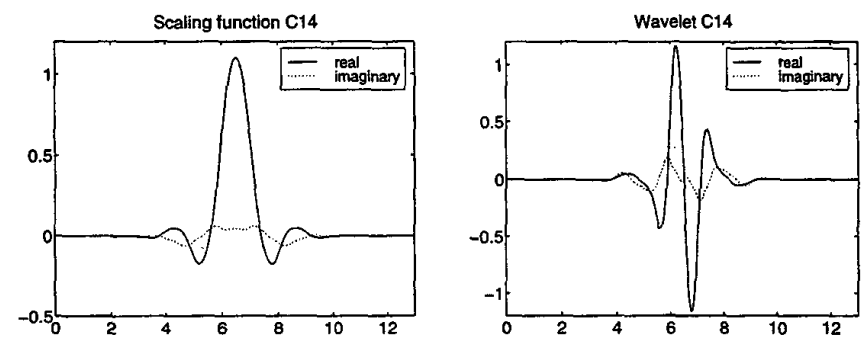

Figure 1: Minimum time localized scaling function and wavelet with 7 vanishing moments. 

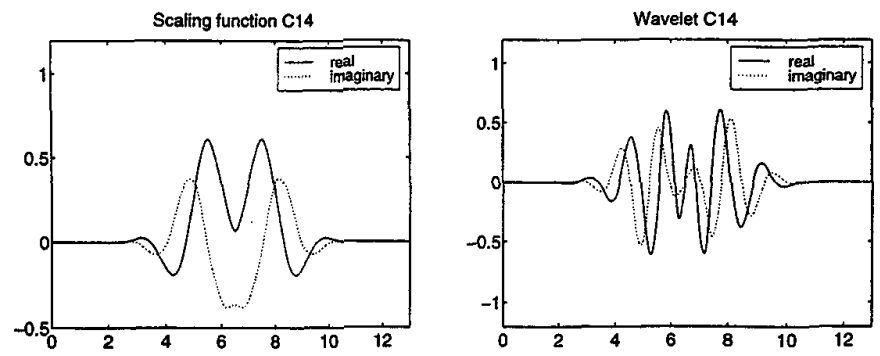

Figure 2: Maximum time localized scaling function and wavelet with 7 vanishing moments.

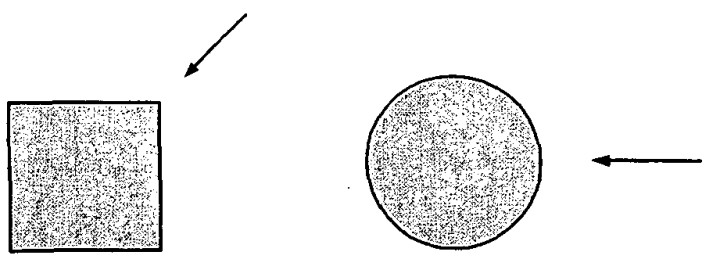

Figure 3: (a)Square cylinder and (b)Circular cylinder


Figure 4: Compression ratio vs. vanishing moment for matrix size $N=512$. Minimum localized complex-valued Daubechies wavelet (Min CD), maximum localized complex-valued Daubechies wavelet (Max CD) and real-valued Daubechies wavelet (RD) are compared for (a)Square cylinder and (b)Circular cylinder 